\title{
DYNAMIC PERFORMANCE OF LAMINATED COMPOSITE PLATES WITHOUT HOLE
}

\author{
Sagar Umatar ${ }^{1}$, Vinayak Kallannavar ${ }^{2}$, Vinayak $\operatorname{Ratan}^{3}$, Anil Shirahatti ${ }^{4}$ \\ ${ }^{1}$ Student, Mechanical Department, Jain College of Engineering, Belagavi, Karnataka, India \\ ${ }^{2}$ Assistant Professor, Mechanical Department, Jain College of Engineering, Belagavi, Karnataka, India \\ ${ }^{3}$ Assistant Professor, Mechanical Department, Jain College of Engineering, Belagavi, Karnataka, India \\ ${ }^{4}$ Professor, Mechanical Department, Jain College of Engineering, Belagavi, Karnataka, India
}

\begin{abstract}
Composite laminates are assembly of several fiber layers, which when combined together will provide the required properties to the composite and yield good weight to strength ratio and stiffness to density ratio. Composite materials are often subjected to vibration used in various fields. In this study, an effort has been made to know the variation of natural frequency of composite plates without hole for three different materials (Glass-Epoxy, Boron-Epoxy and Graphite-Epoxy). Modal analysis is carried out for composite plates for all the three materials without hole by considering various fiber orientations subjected to different boundary conditions. Further, the study was carried out by considering different aspect ratios to understand the variation of natural frequency on composite plate. The analysis was carried out using ANSYS 14.5 simulation tool. It was found out that composite plate had higher natural frequency for [90/45] fiber orientation for all materials.
\end{abstract}

Keywords: Modal analysis, natural frequency, Glass-Epoxy, Boron-Epoxy, Graphite-Epoxy, ANSYS 14.5

\section{INTRODUCTION}

Composite materials are slowly replacing monolithic materials and their alloys in automobile, aeronautical, medical, construction, marine, communications and many other fields. Composite laminates are assembly of several fiber layers, which when combined together will provide the required properties to the composite and yield good weight to strength ratio and stiffness to density ratio. Most commonly used composites are Glass-Epoxy, Boron Epoxy, Graphite Epoxy, Carbon Kevlar-Epoxy etc. Several researches are going on in order to improve the characteristics of the composites so that they can be extensively used in wider number of fields. Kumar et. al. [1] carried out both experimental and finite element (FE) analysis of Glass-Epoxy composite plate under different boundary conditions to determine the corresponding natural frequencies. They observed that fixed-fixed boundary condition has higher frequencies than free-free boundary condition. Itishree et. al. [2] also carried out the experimental and FE analysis of Glass-epoxy composite for different aspect ratios and fiber orientations under free-free boundary condition. It was found that with the increase in aspect ratio, the natural frequencies increased. Swapnil et. al and Sharayu et. al. [3,4] analyzed Glass-epoxy composite under fixed-free boundary condition for different aspect ratios and fiber orientations. They concluded that the natural frequencies increased with increase in aspect ratio and [30/60] orientation had higher frequencies than that of [45/-45] orientation. Chaudari et. al. [5] conducted an experimental and $\mathrm{FE}$ analysis to compare the natural frequencies of triangular shaped composite and metallic plates. It was observed that the metallic plates had higher natural frequencies than that of the composite plates. Chetan et. al. [6] carried out the FE analysis of Glass-epoxy composite to determine the natural frequencies for different number of layers. It was reported that with the decrease in the number of layers the natural frequency also decreased. Nayak et. al. [7] carried out numerical and experimental analysis of Carbon Glass-epoxy composite to determine the natural frequencies and understand the buckling characteristics for different fiber orientations and fiber concentrations. They reported that the buckling characteristics changed with change in the concentration of carbon and glass fibers in the composite. Parida et. al. [8] carried out FE analysis for a composite plate considering it as a beam for various fiber orientations and boundary conditions. It was observed that the natural frequencies increased up to some extent and then remained constant irrespective of the fiber orientations and boundary conditions.

Itishree et. al. [10] carried out experimental and FE analysis on Glass - Epoxy composite plates for different boundary conditions and fiber orientations. It was found that the plate with cantilever boundary condition had the least natural frequency among all boundary condition. Deshmukh et. al. [11] carried out the FE analysis of a sandwiched composite with a core in honey comb structure. They aimed to compare the sandwiched composite and the aluminum plate. In the practical scenario, composite plates are used with cutout at suitable locations are used. Hence, in past few decades emphasis is laid on understanding the behavior of composite plates with geometric nonlinearities. 
In present study, an attempt has been made to understand the modal behavior of composite plates for different fiber orientations, aspect ratios and geometric nonlinearities by considering different boundary conditions. The natural frequencies obtained from FE simulation were analyzed to estimate the most suitable composite material for required applications.

\section{FINITE ELEMENT ANALYSIS}

The finite element analysis of composite plate without hole is carried out in ANSYS 14.5 simulation tool. The shell element 8 Node281 type was used to carry out the analysis. The plate with the dimensions of $0.15 \mathrm{~m} \mathrm{X} 0.11 \mathrm{~m}$ was analyzed with the overall thickness of $2.1 \mathrm{~mm}$. The composite laminate is considered to have 7 layers with the thickness of each layer to be $0.3 \mathrm{~mm}$. The plate is meshed to get 540 number of elements and 1715 number of nodes.

The plate is analyzed for two boundary conditions namely, both ends fixed and cantilever condition. Both ends fixed boundary condition includes fixing the two opposite sides while cantilever condition includes fixing the first side of the plate as shown in Fig. 1.

Table 1 Material properties considered for composite plate without hole $[1,12]$

\begin{tabular}{|l|l|l|l|}
\hline Property & $\begin{array}{l}\text { Glass- } \\
\text { Epoxy }\end{array}$ & $\begin{array}{l}\text { Boron- } \\
\text { Epoxy }\end{array}$ & $\begin{array}{l}\text { Graphite- } \\
\text { Epoxy }\end{array}$ \\
\hline $\boldsymbol{\rho}\left(\mathrm{kg} / \mathrm{m}^{3}\right)$ & 2400 & 1960 & 1580 \\
\hline $\mathbf{E}_{\mathbf{x}}(\mathrm{GPa})$ & 46.2 & 204 & 181 \\
\hline $\mathbf{E}_{\mathbf{y}}(\mathrm{GPa})$ & 14.7 & 18.5 & 10.30 \\
\hline $\mathbf{E}_{\mathbf{z}}(\mathrm{GPa})$ & 14.7 & 18.5 & 10.30 \\
\hline $\mathbf{v}_{\mathbf{x y}}$ & 0.31 & 0.23 & 0.28 \\
\hline $\mathbf{v}_{\mathbf{y z}}$ & 0.31 & 0.23 & 0.28 \\
\hline $\mathbf{v}_{\mathbf{x z}}$ & 0.41 & 0.29 & 0.35 \\
\hline $\mathbf{G}_{\mathbf{x y}}(\mathrm{GPa})$ & 5.35 & 5.59 & 7.17 \\
\hline $\mathbf{G}_{\mathbf{y z}}(\mathrm{GPa})$ & 5.35 & 5.59 & 7.17 \\
\hline $\mathbf{G}_{\mathbf{x z}}(\mathrm{GPa})$ & 5.22 & 5.45 & 7.34 \\
\hline
\end{tabular}

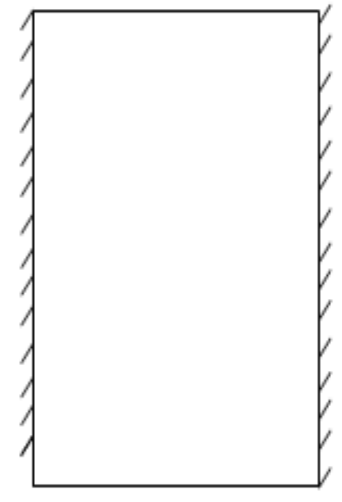

(a)

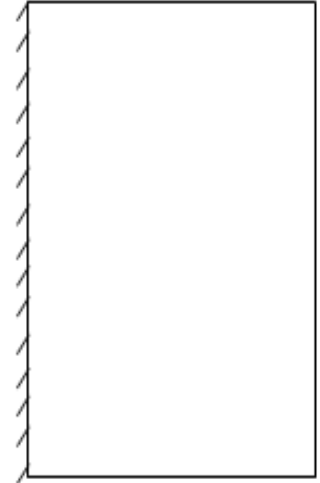

(b)
Fig 1: Boundary condition (a) Both ends fixed (b) Cantilever

\section{RESULTS AND DISCUSSION}

Modal analysis was carried out for the Glass-Epoxy composite plate (without hole) under cantilever and both end fixed boundary conditions using ANSYS simulation tool. The FEM results obtained were compared with the published results [1] and the results were in good agreement with each other.

Table 2: Comparison between published and FEM results of composite plate without hole

\begin{tabular}{|l|l|l|l|}
\hline $\begin{array}{l}\text { Boundary } \\
\text { Condition }\end{array}$ & $\begin{array}{l}\text { Mode } \\
\text { Number }\end{array}$ & $\begin{array}{l}\text { Published } \\
\text { Results [1] }\end{array}$ & $\begin{array}{l}\text { FEM } \\
\text { Results }\end{array}$ \\
\hline Cantilever & 1 & 89.323 & 89.319 \\
condition & 2 & 149.88 & 149.97 \\
& 3 & 450.77 & 450.71 \\
\hline Both ends & 1 & 566.40 & 566.23 \\
fixed & 2 & 603.92 & 603.81 \\
condition & 3 & 815.67 & 814.41 \\
\hline
\end{tabular}

Further, the study was carried out to understand the effect on natural frequency for composite plate without hole for three different composite materials (i.e. Glass-Epoxy, Boron-Epoxy and Graphite-Epoxy) for cantilever and both ends fixed boundary conditions with different fiber orientations.

The study was then carried out by considering different aspect ratios such as length to thickness, length to width and width to thickness for both cantilever and both ends fixed boundary condition for glass/epoxy composite plate. In all the cases natural frequency decreased with increase in aspect ratio and also, the natural frequency of both ends fixed boundary condition was more compared to that of cantilever boundary condition.

The natural frequencies obtained for both-ends fixed Boundary Condition for Glass-Epoxy, Boron-epoxy and Graphite-Epoxy materials are listed in Table 3, Table 4 \& Table 5 respectively. 
Table 3: Glass-Epoxy (Both ends fixed boundary condition)

\begin{tabular}{|l|l|l|l|l|l|l|l|l|l|l|}
\hline ORIENTATIONS & $\mathbf{1}$ & $\mathbf{2}$ & $\mathbf{3}$ & $\mathbf{4}$ & $\mathbf{5}$ & $\mathbf{6}$ & $\mathbf{7}$ & $\mathbf{8}$ & $\mathbf{9}$ & $\mathbf{1 0}$ \\
\hline$[\mathbf{0 / 3 0}]_{7}$ & 452.76 & 511.8 & 789.27 & 1244.6 & 1326.9 & 1418.7 & 1637.5 & 2208.5 & 2431.5 & 2461 \\
\hline$[\mathbf{0 / 6 0}]_{7}$ & 514.22 & 564 & 812.55 & 1385.9 & 1422.7 & 1479.4 & 1750.5 & 2255.6 & 2381.3 & 2758.5 \\
\hline$[\mathbf{3 0 / 6 0}]_{7}$ & 524.61 & 590.27 & 846.55 & 1328.9 & 1447.6 & 1530.3 & 1878.2 & 2052.2 & 2472.8 & 2824.8 \\
\hline$[\mathbf{3 0 / 9 0}]_{7}$ & 578.34 & 637.34 & 869.27 & 1343.7 & 1586.7 & 1666.7 & 1963 & 2077.4 & 2502.2 & 2968.5 \\
\hline$[\mathbf{6 0 / 9 0}]_{7}$ & 688.72 & 730.24 & 915.58 & 1284 & 1854.8 & 1887.4 & 1956.6 & 2170 & 2573.1 & 2684.9 \\
\hline$[\mathbf{3 0 / 6 0 / 9 0 / 0 / 9 0 / 6 0 / 3 0}]$ & 552.7 & 614.34 & 858.64 & 1331.1 & 1520.8 & 1599.5 & 1924.6 & 2050.4 & 2487 & 2908.6 \\
\hline$[\mathbf{0 / 3 0 / 6 0 / 9 0 / 6 0 / 3 0 / 0}]$ & 472.7 & 531.41 & 801.96 & 1298.5 & 1380.5 & 1410 & 1689.1 & 2227.2 & 2434.9 & 2539.1 \\
\hline$[\mathbf{0 / 4 5}]_{7}$ & 476.04 & 534.76 & 805.74 & 1307.4 & 1389.4 & 1413.1 & 1698.1 & 2242.1 & 2425.5 & 2559.3 \\
\hline$[\mathbf{3 0 / 4 5}]_{7}$ & 487.54 & 563.05 & 838.27 & 1324.7 & 1358.8 & 1445.9 & 1832 & 2062.4 & 2473.6 & 2636.2 \\
\hline$[\mathbf{6 0 / 4 5}]_{7}$ & 611.8 & 660.21 & 882.74 & 1290.5 & 1686.3 & 1725 & 1934.7 & 2033.2 & 2507.2 & 2744.4 \\
\hline$[\mathbf{9 0 / 4 5}]_{7}$ & 724.89 & 764.71 & 928.71 & 1284.2 & 1876.8 & 1983.4 & 2037 & 2234.6 & 2599 & 2709 \\
\hline$[\mathbf{3 0 / 6 0 / 9 0 / 4 5 / 9 0 / 6 0 / 3 0}]$ & 552.89 & 614.56 & 858.81 & 1330.6 & 1521.3 & 1600 & 1925.3 & 2048.8 & 2487.3 & 2906.6 \\
\hline$[\mathbf{3 0 / 4 5 / 6 0 / 9 0 / 6 0 / 4 5 / 3 0 ]}]$ & 503.65 & 575.52 & 843.7 & 1329.8 & 1393.7 & 1483.2 & 1856 & 2053.9 & 2475.6 & 2718.5 \\
\hline$[\mathbf{4 5 / 9 0}]_{7}$ & 619.45 & 676.44 & 899.72 & 1320.9 & 1700.6 & 1769.1 & 1975.5 & 2068.9 & 2561.2 & 2826 \\
\hline
\end{tabular}

Table 4: Boron-Epoxy (Both ends fixed boundary condition)

\begin{tabular}{|l|l|l|l|l|l|l|l|l|l|l|}
\hline ORIENTATIONS & $\mathbf{1}$ & $\mathbf{2}$ & $\mathbf{3}$ & $\mathbf{4}$ & $\mathbf{5}$ & $\mathbf{6}$ & $\mathbf{7}$ & $\mathbf{8}$ & $\mathbf{9}$ & $\mathbf{1 0}$ \\
\hline$[\mathbf{0 / 3 0}]_{7}$ & 581.47 & 687.78 & 1319.6 & 1598.1 & 1758 & 2316.1 & 2854.7 & 3114.4 & 3291.1 & 3538 \\
\hline$[\mathbf{0 / 6 0}]_{7}$ & 855.38 & 901.6 & 1396.9 & 2319.4 & 2390.6 & 2610 & 2930.1 & 3750.6 & 4527.6 & 4556.7 \\
\hline$[\mathbf{3 0 / 6 0}]_{7}$ & 872 & 954.82 & 1390.4 & 2114.9 & 2426.4 & 2466.9 & 3162.4 & 3194.6 & 4191.9 & 4270 \\
\hline$[\mathbf{3 0 / 9 0}]_{7}$ & 1101.8 & 1196.1 & 1581.6 & 2374.3 & 2985.4 & 3104.5 & 3552.7 & 3626.7 & 4621.3 & 4896.8 \\
\hline$[\mathbf{6 0 / 9 0}]_{7}$ & 1431.6 & 1461.4 & 1720.4 & 2172.3 & 2877.3 & 3722.9 & 3855.2 & 3950.5 & 4263.4 & 4681 \\
\hline$[\mathbf{3 0 / 6 0 / 9 0 / 0 / 9 0 / 6 0 / 3 0}]$ & 988.23 & 1067.9 & 1474.3 & 2216.9 & 2699.7 & 2755.6 & 3327.6 & 3373 & 4375.3 & 4516 \\
\hline$[\mathbf{0 / 3 0 / 6 0 / 9 0 / 6 0 / 3 0 / 0}]$ & 678.26 & 771.79 & 1356 & 1859.4 & 1997 & 2445.3 & 2876.1 & 3533.8 & 3724.8 & 3756.7 \\
\hline$[\mathbf{0 / 4 5}]_{7}$ & 689.29 & 777.8 & 1364.8 & 1891.9 & 2018.4 & 2467.4 & 2881.1 & 3544.3 & 3804.3 & 3813.9 \\
\hline$[\mathbf{3 0 / 4 5}]_{7}$ & 713.41 & 841.6 & 1338.45 & 1935.1 & 2140.8 & 2149.7 & 2978.9 & 3107.2 & 3789.9 & 3961.8 \\
\hline$[\mathbf{6 0 / 4 5}]_{7}$ & 1143.3 & 1161.3 & 1515.7 & 2037.5 & 2746.1 & 3075.4 & 3200.7 & 3620.9 & 4005 & 4209.4 \\
\hline$[\mathbf{9 0 / 4 5}]_{7}$ & 1581.9 & 1628.5 & 1830.8 & 2267.4 & 3019.6 & 4088.7 & 4244.3 & 4298.5 & 4560.8 & 4981.3 \\
\hline$[\mathbf{3 0 / 6 0 / 9 0 / 4 5 / 9 0 / 6 0 / 3 0 ]}$ & 988.65 & 1068.2 & 1473.9 & 2213.4 & 2700.2 & 2755.5 & 3320.6 & 3373.2 & 4371.5 & 4506.4 \\
\hline$[\mathbf{3 0 / 4 5 / 6 0 / 9 0 / 6 0 / 4 5 / 3 0 ]}$ & 782.12 & 890.25 & 1363.8 & 2046 & 2232.8 & 2279.4 & 3058.3 & 3135 & 4009.3 & 4133.8 \\
\hline$[\mathbf{4 5 / 9 0}]_{7}$ & 1223.8 & 1301.9 & 1663.2 & 2271.8 & 3139.2 & 3302 & 3431.4 & 3881.9 & 4397.2 & 4668.1 \\
\hline
\end{tabular}

Table 5: Graphite-Epoxy (Both ends fixed boundary condition)

\begin{tabular}{|l|l|l|l|l|l|l|l|l|l|l|}
\hline ORIENTATIONS & $\mathbf{1}$ & $\mathbf{2}$ & $\mathbf{3}$ & $\mathbf{4}$ & $\mathbf{5}$ & $\mathbf{6}$ & $\mathbf{7}$ & $\mathbf{8}$ & $\mathbf{9}$ & $\mathbf{1 0}$ \\
\hline$[\mathbf{0 / 3 0}]_{\mathbf{7}}$ & 521.16 & 655.15 & 1343.6 & 1439 & 1655.1 & 2283.4 & 2814.5 & 2972.5 & 3079.4 & 3488.3 \\
\hline$[\mathbf{0 / 6 0}]_{7}$ & 854.26 & 912.26 & 1453 & 2332.1 & 2414.8 & 2696.2 & 3052.3 & 3881.8 & 4587.2 & 4613 \\
\hline$[\mathbf{3 0 / 6 0}]_{7}$ & 895.04 & 955.9 & 1372.7 & 2064.4 & 2483.2 & 2513.3 & 3135.4 & 3191.5 & 4118.5 & 4202.9 \\
\hline$[\mathbf{3 0 / 9 0}]_{7}$ & 1149.7 & 1245.6 & 1639.2 & 2450.2 & 3135.1 & 3249.7 & 3666.8 & 3795 & 4832.5 & 5042.2 \\
\hline$[\mathbf{6 0 / 9 0}]_{7}$ & 1522.8 & 1553.2 & 1807 & 2227.2 & 2869.7 & 3716.2 & 4125.8 & 4153.7 & 4544.3 & 4788.4 \\
\hline$[\mathbf{3 0 / 6 0 / 9 0 / 0 / 9 0 / 6 0 / 3 0}]$ & 1026.6 & 1094.4 & 1491.4 & 2223.4 & 2823.4 & 2830.6 & 3354.8 & 3467.1 & 4478.2 & 4495.2 \\
\hline$[\mathbf{0 / 3 0 / 6 0 / 9 0 / 6 0 / 3 0 / 0}]$ & 645.82 & 755.26 & 1389.2 & 1779.4 & 1945.3 & 2450.8 & 3004.3 & 3427.4 & 3648.8 & 3726.6 \\
\hline$[\mathbf{0 / 4 5}]_{7}$ & 660.73 & 764.19 & 1399.5 & 1824.1 & 1975.5 & 2477.3 & 3009.4 & 3475.8 & 3718.3 & 3807.4 \\
\hline$[\mathbf{3 0 / 4 5}]_{7}$ & 719.34 & 817.05 & 1286.2 & 1885.6 & 2119 & 2140.5 & 2942.7 & 2943 & 3779.7 & 3810.3 \\
\hline$[\mathbf{6 0 / 4 5}]_{7}$ & 1200.6 & 1209.8 & 1544.7 & 1999.1 & 2652.3 & 3174.2 & 3354.8 & 3684.4 & 3996.5 & 4253.4 \\
\hline$[\mathbf{9 0 / 4 5}]_{7}$ & 1674.6 & 1727.4 & 1938.5 & 2366.3 & 3079.6 & 4093 & 4530.7 & 4595.5 & 4874.5 & 5247.5 \\
\hline$[\mathbf{3 0 / 6 0 / 9 0 / 4 5 / 9 0 / 6 0 / 3 0}]$ & 1027.4 & 1094.9 & 1490.9 & 2219.2 & 2825.2 & 2830.5 & 3347.9 & 3467.8 & 4473.7 & 4484.6 \\
\hline$[\mathbf{3 0 / 4 5 / 6 0 / 9 0 / 6 0 / 4 5 / 3 0 ]}]$ & 797.91 & 878.4 & 1327.3 & 1979.5 & 2279.8 & 2290.1 & 3019.6 & 3053.3 & 3954.5 & 3992.6 \\
\hline$[\mathbf{4 5 / 9 0}]_{7}$ & 1295.1 & 1370 & 1724.5 & 2318.8 & 3175.2 & 3517.3 & 3638.1 & 4052.6 & 4455.3 & 4853.1 \\
\hline
\end{tabular}


From the results it is evident that, for both-ends fixed boundary condition the $[90 / 45]_{7}$ fiber orientation has higher natural frequency value for all the 3 materials chosen for most of the modes.
The natural frequencies obtained for Cantilever Boundary Condition for Glass-Epoxy, Boron-epoxy and GraphiteEpoxy materials are listed in Table 6, Table $7 \&$ Table 8 respectively.

Table 6: Glass-Epoxy (cantilever boundary condition)

\begin{tabular}{|l|l|l|l|l|l|l|l|l|l|l|}
\hline ORIENTATIONS & $\mathbf{1}$ & $\mathbf{2}$ & $\mathbf{3}$ & $\mathbf{4}$ & $\mathbf{5}$ & $\mathbf{6}$ & $\mathbf{7}$ & $\mathbf{8}$ & $\mathbf{9}$ & $\mathbf{1 0}$ \\
\hline$[\mathbf{0 / 3 0}]_{7}$ & 71.141 & 150.14 & 431.91 & 488.23 & 581.58 & 949.92 & 1171.2 & 1246.1 & 1377.3 & 1609.1 \\
\hline$[\mathbf{0 / 6 0}]_{7}$ & 80.308 & 155.7 & 454.26 & 512.72 & 623.43 & 970.94 & 1118.5 & 1409.1 & 1516.3 & 1609.7 \\
\hline$[\mathbf{3 0 / 6 0}]_{7}$ & 81.461 & 171.96 & 409.88 & 519.53 & 671 & 888.26 & 1112 & 1432.8 & 1480.8 & 1601.4 \\
\hline$[\mathbf{3 0 / 9 0}]_{7}$ & 90.946 & 172.04 & 417.42 & 522.68 & 701.71 & 896.06 & 1123.1 & 1489.8 & 1584.9 & 1714.3 \\
\hline$[\mathbf{6 0 / 9 0}]_{7}$ & 106.72 & 183.92 & 387.83 & 670.09 & 743.67 & 836.84 & 1089.9 & 1413 & 1559 & 1892.1 \\
\hline$[\mathbf{3 0 / 6 0 / 9 0 / 0 / 9 0 / 6 0 / 3 0 ]}]$ & 86.102 & 173.76 & 412.1 & 545.35 & 687.59 & 889.19 & 1112.7 & 1485.5 & 1507.7 & 1659 \\
\hline$[\mathbf{0 / 3 0 / 6 0 / 9 0 / 6 0 / 3 0 / 0}]$ & 74.167 & 154.51 & 442.26 & 490.61 & 601.69 & 962.63 & 1149.5 & 1299.8 & 1430 & 1607.6 \\
\hline$[\mathbf{0 / 4 5}]_{7}$ & 74.515 & 155.98 & 443.97 & 492.56 & 604.27 & 972.04 & 1141.3 & 1308.3 & 1439.3 & 1619.2 \\
\hline$[\mathbf{3 0 / 4 5}]_{7}$ & 75.785 & 170.62 & 403.31 & 491.71 & 653.62 & 886.57 & 1121.9 & 1334.8 & 1478.2 & 1531.4 \\
\hline$[\mathbf{6 0 / 4 5}]_{7}$ & 93.021 & 182.03 & 394.45 & 588.85 & 708.71 & 842.22 & 1078 & 1433.1 & 1552.2 & 1714.9 \\
\hline$[\mathbf{9 0 / 4 5}]_{7}$ & 114.32 & 181.1 & 385.52 & 713.92 & 767.45 & 844.47 & 1090.2 & 1427.7 & 1569.8 & 1989.3 \\
\hline$[\mathbf{3 0 / 6 0 / 9 0 / 4 5 / 9 0 / 6 0 / 3 0 ]}]$ & 86.128 & 173.83 & 411.8 & 545.5 & 687.79 & 888.27 & 1112.8 & 1484.4 & 1508 & 1659.4 \\
\hline$[\mathbf{3 0 / 4 5 / 6 0 / 9 0 / 6 0 / 4 5 / 3 0 ]}]$ & 78.193 & 172.25 & 405.66 & 503.17 & 661.9 & 885.72 & 1117 & 1377 & 1479.3 & 1562.3 \\
\hline$[\mathbf{4 5 / 9 0}]_{7}$ & 96.622 & 183.72 & 402.88 & 607.75 & 726.88 & 858 & 1119.4 & 1454.9 & 1640.5 & 1746.6 \\
\hline
\end{tabular}

Table 7: Boron-Epoxy (cantilever boundary condition)

\begin{tabular}{|l|l|l|l|l|l|l|l|l|l|l|}
\hline ORIENTATIONS & $\mathbf{1}$ & $\mathbf{2}$ & $\mathbf{3}$ & $\mathbf{4}$ & $\mathbf{5}$ & $\mathbf{6}$ & $\mathbf{7}$ & $\mathbf{8}$ & $\mathbf{9}$ & $\mathbf{1 0}$ \\
\hline$[\mathbf{0 / 3 0}]_{7}$ & 90.523 & 222.41 & 566.91 & 781.29 & 1015.3 & 1503.9 & 1604.4 & 1872.3 & 2462.8 & 2581.4 \\
\hline$[\mathbf{0 / 6 0}]_{7}$ & 126.04 & 241.33 & 813.26 & 884.13 & 1046.4 & 1550.1 & 2287.8 & 2360.7 & 2544.9 & 2706.8 \\
\hline$[\mathbf{3 0 / 6 0}]_{7}$ & 129.91 & 269.4 & 641.52 & 820.97 & 1140.2 & 13225.2 & 1891.8 & 2149 & 2363.7 & 2678.1 \\
\hline$[\mathbf{3 0 / 9 0}]_{7}$ & 174.66 & 283.93 & 708.32 & 1086.7 & 1304.5 & 1428 & 2142.5 & 2321.7 & 2989.9 & 3185.3 \\
\hline$[\mathbf{6 0 / 9 0}]_{7}$ & 217.4 & 338.61 & 615.77 & 1101.2 & 1340.2 & 1582 & 1854.7 & 2047.6 & 2607.5 & 2910.6 \\
\hline$[\mathbf{3 0 / 6 0 / 9 0 / 0 / 9 0 / 6 0 / 3 0 ]}$ & 150.63 & 283.67 & 668.79 & 943.85 & 1217.5 & 1375.2 & 1991.9 & 2241.4 & 2639.5 & 2878.3 \\
\hline$[\mathbf{0 / 3 0 / 6 0 / 9 0 / 6 0 / 3 0 / 0}]$ & 104.26 & 238.83 & 657.66 & 827.04 & 1027.8 & 1542.2 & 1860.8 & 2098.5 & 2485.4 & 2597.9 \\
\hline$[\mathbf{0 / 4 5}]_{7}$ & 103.64 & 242.43 & 662.76 & 840.32 & 1020 & 1544.9 & 1887.3 & 2137.9 & 2485.1 & 2594.3 \\
\hline$[\mathbf{3 0 / 4 5}]_{7}$ & 106.55 & 260.68 & 579.73 & 712.44 & 1051.3 & 1303.2 & 1781.8 & 1937.8 & 2144.2 & 2513.5 \\
\hline$[\mathbf{6 0 / 4 5}]_{7}$ & 158.99 & 307.68 & 599.54 & 978.4 & 1092.4 & 1403.7 & 1735 & 1994.2 & 2377.4 & 2789.5 \\
\hline$[\mathbf{9 0 / 4 5}]_{7}$ & 252.95 & 340.56 & 618.86 & 1172.8 & 1561.1 & 1692.6 & 1980.4 & 2111.5 & 2755.2 & 3040.2 \\
\hline$[\mathbf{3 0 / 6 0 / 9 0 / 4 5 / 9 0 / 6 0 / 3 0 ]}]$ & 150.69 & 283.54 & 666.92 & 944.07 & 1216 & 1372.4 & 1988.9 & 2235.7 & 2638.7 & 2875.6 \\
\hline$[\mathbf{3 0 / 4 5 / 6 0 / 9 0 / 6 0 / 4 5 / 3 0}]$ & 116.51 & 267.95 & 610.31 & 752.23 & 1085.3 & 1314.6 & 1823.9 & 2077.5 & 2186.7 & 2580.9 \\
\hline$[\mathbf{4 5 / 9 0}]_{7}$ & 190.19 & 330.61 & 651.75 & 1179.5 & 1199.6 & 1484.1 & 1999.1 & 2174.3 & 2894.4 & 3167 \\
\hline
\end{tabular}

From the results it is evident that, for cantilever boundary Condition the $[90 / 45]_{7}$ fiber orientation has higher natural frequency value for all the 3 materials chosen for most of the modes.

\section{Aspect Ratios}

Study was further extended to understand the effect of aspect ratio on the natural frequencies of the composite plate (without hole) by considering Glass-Epoxy material for simulations of different Boundary Conditions.

Natural frequencies for varying Length:Thickness ratios were studied by keeping the plate length constant as $0.15 \mathrm{~m}$ and varying the thickness. The results for Both Ends Fixed
\& Cantilever boundary condition are listed in Table 9 and table 10 respectively.

The obtained results are plotted in fig 2 and fig 3 for both ends fixed and cantilever boundary conditions and it was observed that the magnitude of natural frequencies decreased with increase in Length:Thickness ratio. 
Table 8: Graphite-Epoxy (cantilever boundary condition)

\begin{tabular}{|l|l|l|l|l|l|l|l|l|l|l|}
\hline ORIENTATIONS & $\mathbf{1}$ & $\mathbf{2}$ & $\mathbf{3}$ & $\mathbf{4}$ & $\mathbf{5}$ & $\mathbf{6}$ & $\mathbf{7}$ & $\mathbf{8}$ & $\mathbf{9}$ & $\mathbf{1 0}$ \\
\hline$[\mathbf{0 / 3 0}]_{7}$ & 80.18 & 231.06 & 507.8 & 774.35 & 1061.5 & 1448 & 1503.6 & 1840.2 & 2452.7 & 2694.4 \\
\hline$[\mathbf{0 / 6 0}]_{7}$ & 125.25 & 256.06 & 812.55 & 919.95 & 1086.5 & 1622.3 & 2303.1 & 2464.4 & 2613.3 & 2794.6 \\
\hline$[\mathbf{3 0 / 6 0}]_{7}$ & 128.29 & 261.29 & 634.85 & 818.19 & 1160.2 & 1280.9 & 1872.6 & 2064.4 & 2445.4 & 2633.2 \\
\hline$[\mathbf{3 0 / 9 0}]_{7}$ & 180.66 & 294.79 & 734.87 & 1127.3 & 1369 & 1468.1 & 2235.8 & 2391.2 & 3124.1 & 3304.7 \\
\hline$[\mathbf{6 0 / 9 0}]_{7}$ & 229.03 & 350.82 & 614.99 & 1062.7 & 1426.4 & 1626.7 & 1806.8 & 2108.5 & 2624.4 & 2786.5 \\
\hline$[\mathbf{3 0 / 6 0 / 9 0 / 0 / 9 0 / 6 0 / 3 0}]$ & 152.82 & 282.51 & 674 & 964.08 & 1254.1 & 1367.3 & 2023.9 & 2218.1 & 2742.6 & 2891.3 \\
\hline$[\mathbf{0 / 3 0 / 6 0 / 9 0 / 6 0 / 3 0 / 0}]$ & 97.859 & 247.9 & 625.26 & 833.63 & 1070.6 & 1564.8 & 1781.4 & 2095.8 & 2564.7 & 2655.3 \\
\hline$[\mathbf{0 / 4 5}]_{7}$ & 97.768 & 250.98 & 634.26 & 847.77 & 1062.6 & 1563.9 & 1823.8 & 2145.3 & 2591.1 & 2620.1 \\
\hline$[\mathbf{3 0 / 4 5}]_{7}$ & 103.04 & 248.9 & 593.66 & 668.01 & 1058.2 & 1236.8 & 1742.6 & 1871.5 & 2126.9 & 2487.3 \\
\hline$[\mathbf{6 0 / 4 5}]_{7}$ & 162.93 & 305.11 & 575.12 & 1005.9 & 1055.1 & 1436.5 & 1718.4 & 1906.6 & 2395 & 2614.1 \\
\hline$[\mathbf{9 0 / 4 5}]_{7}$ & 265.72 & 361.73 & 636.27 & 1163 & 1647.4 & 1784.9 & 1953.8 & 2217.2 & 2866.2 & 2980 \\
\hline$[\mathbf{3 0 / 6 0 / 9 0 / 4 5 / 9 0 / 6 0 / 3 0}]$ & 152.88 & 282.18 & 671.73 & 964.33 & 1251.4 & 1364.9 & 2019 & 2212.3 & 2741.9 & 2885.2 \\
\hline$[\mathbf{3 0 / 4 5 / 6 0 / 9 0 / 6 0 / 4 5 / 3 0 ]}]$ & 114.16 & 256.99 & 613.23 & 729.68 & 1099 & 1257.3 & 1796.7 & 1972.8 & 2244.9 & 2549 \\
\hline$[\mathbf{4 5 / 9 0}]_{7}$ & 198.95 & 335.77 & 657.15 & 1202.9 & 1244.9 & 1537 & 2009.5 & 2206.8 & 2942.9 & 3151.6 \\
\hline
\end{tabular}

Table 9: Both Ends Fixed

\begin{tabular}{|l|l|l|l|l|l|l|l|l|l|l|}
\hline $\begin{array}{l}\text { L:T } \\
\text { Ratios }\end{array}$ & $\mathbf{1}$ & $\mathbf{2}$ & $\mathbf{3}$ & $\mathbf{4}$ & $\mathbf{5}$ & $\mathbf{6}$ & $\mathbf{7}$ & $\mathbf{8}$ & $\mathbf{9}$ & $\mathbf{1 0}$ \\
\hline $\mathbf{2 5}$ & 1565.2 & 1663.8 & 2236.5 & 3739.6 & 4133.2 & 4262.1 & 4794.5 & 6001 & 6178.8 & 6489.5 \\
\hline $\mathbf{5 0}$ & 804.98 & 857.9 & 1156.2 & 1942.9 & 2193.6 & 2265.4 & 2556.7 & 3212.4 & 3261.9 & 4238.9 \\
\hline $\mathbf{7 5}$ & 539.5 & 575.35 & 776.09 & 1305.5 & 1479.8 & 1528.8 & 1727.1 & 2172.9 & 2198.9 & 2883.3 \\
\hline $\mathbf{1 0 0}$ & 405.45 & 432.52 & 583.66 & 982.18 & 1114.7 & 1151.8 & 1301.8 & 1638.7 & 1656.1 & 2178.6 \\
\hline $\mathbf{1 2 5}$ & 323.82 & 345.5 & 466.34 & 784.89 & 891.25 & 921.06 & 1041.2 & 1311.1 & 1324.2 & 1744.4 \\
\hline
\end{tabular}

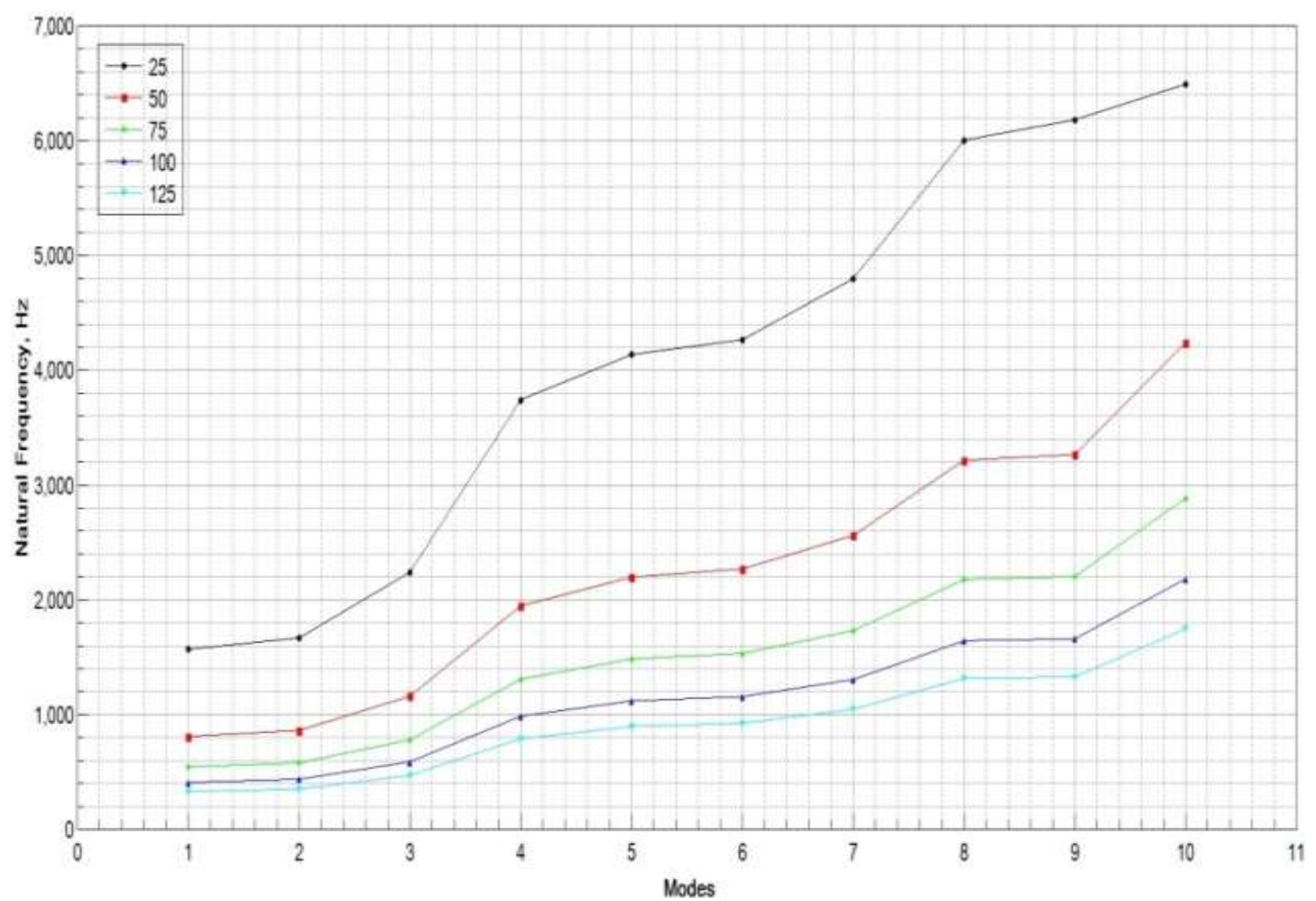

Fig 2: Variation of natural frequency with aspect ratio for both ends fixed condition 
Table 10: Cantilever

\begin{tabular}{|l|l|l|l|l|l|l|l|l|l|l|}
\hline $\begin{array}{l}\text { L:T } \\
\text { Ratios }\end{array}$ & $\mathbf{1}$ & $\mathbf{2}$ & $\mathbf{3}$ & $\mathbf{4}$ & $\mathbf{5}$ & $\mathbf{6}$ & $\mathbf{7}$ & $\mathbf{8}$ & $\mathbf{9}$ & $\mathbf{1 0}$ \\
\hline $\mathbf{2 5}$ & 254.16 & 422.14 & 1267 & 1562.4 & 1789.6 & 2610.6 & 2884.5 & 3054.2 & 4185.3 & 4206.1 \\
\hline $\mathbf{5 0}$ & 127.53 & 213.65 & 642.16 & 797.69 & 917.85 & 1349.4 & 1567.7 & 2183.9 & 2203.9 & 2327 \\
\hline $\mathbf{7 5}$ & 85.067 & 142.86 & 429.34 & 533.84 & 615.13 & 906.25 & 1050.5 & 1469.4 & 1482.7 & 1566.7 \\
\hline $\mathbf{1 0 0}$ & 63.822 & 107.31 & 322.44 & 400.99 & 462.36 & 681.82 & 789.51 & 1106.2 & 1115.8 & 1179.5 \\
\hline $\mathbf{1 2 5}$ & 50.932 & 85.692 & 257.45 & 320.18 & 369.33 & 544.92 & 630.58 & 884.33 & 891.76 & 942.85 \\
\hline
\end{tabular}

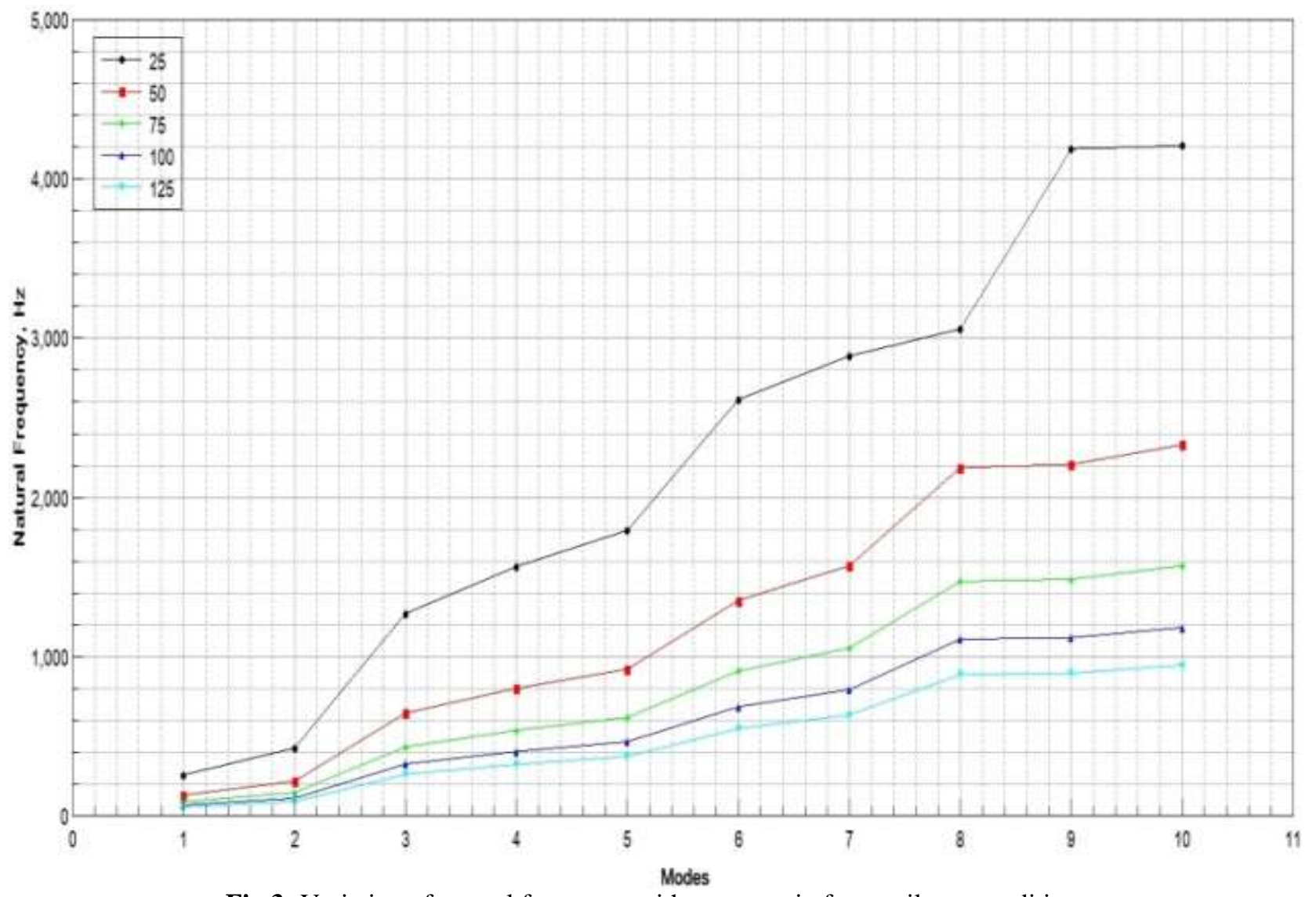

Fig 3: Variation of natural frequency with aspect ratio for cantilever condition

Similarly the natural frequencies for varying Length:Width ratios are obtained by considering width as constant of $0.11 \mathrm{~m}$ and varying the length. The results obtained for Both Ends Fixed and Cantilever boundary condition are listed in Table 11 \& Table 12 respectively.
The obtained results are plotted in fig 4 and fig 5 for both ends fixed and cantilever boundary conditions and it was observed that with the increase in length:width ratio the magnitude of natural frequencies also increased.

Table 11: Both Ends Fixed

\begin{tabular}{|l|l|l|l|l|l|l|l|l|l|l|}
\hline $\mathbf{L : ~} \mathbf{W}$ & $\mathbf{1}$ & $\mathbf{2}$ & $\mathbf{3}$ & $\mathbf{4}$ & $\mathbf{5}$ & $\mathbf{6}$ & $\mathbf{7}$ & $\mathbf{8}$ & $\mathbf{9}$ & $\mathbf{1 0}$ \\
\hline $\mathbf{0 . 5}$ & 564.84 & 796.36 & 1547.5 & 1891.7 & 3011.8 & 3102 & 3403.9 & 3929.7 & 4935.4 & 5285.4 \\
\hline $\mathbf{1}$ & 565.84 & 633.67 & 1088.2 & 1551 & 1645.4 & 2061.8 & 2220.7 & 3019.5 & 3044.4 & 3122.7 \\
\hline $\mathbf{1 . 5}$ & 566.36 & 597.62 & 763.93 & 1204.8 & 1552.7 & 1595.2 & 1763.8 & 1965.4 & 2135 & 2785.7 \\
\hline $\mathbf{2}$ & 566.68 & 584.44 & 667.02 & 880.16 & 1271.7 & 1553.9 & 1577.6 & 1668.4 & 1843.9 & 1854.8 \\
\hline $\mathbf{2 . 5}$ & 566.9 & 578.27 & 627.16 & 746.47 & 971.14 & 1314.4 & 1554.7 & 1569.7 & 1626.4 & 1737.2 \\
\hline
\end{tabular}




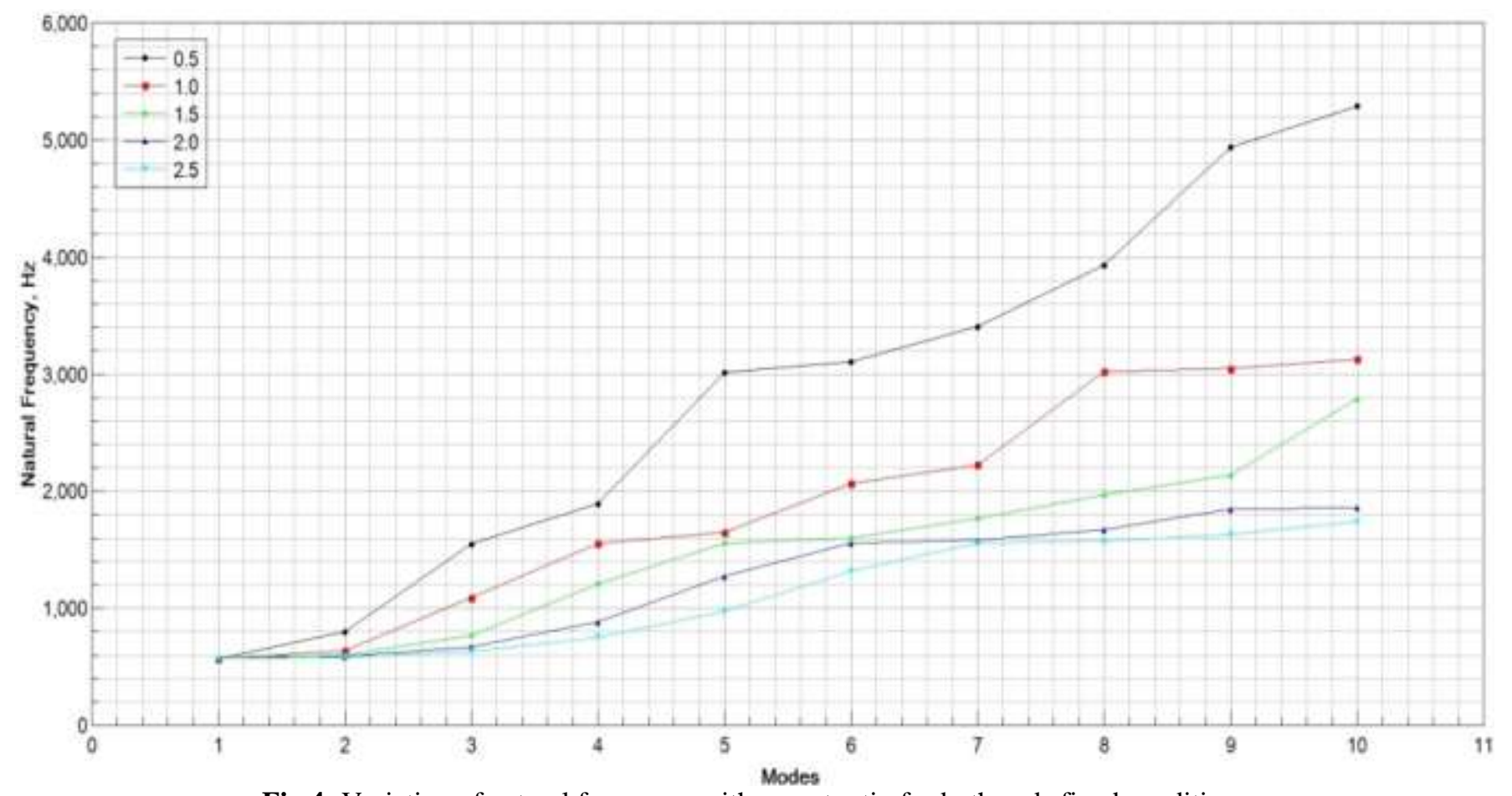

Fig 4: Variation of natural frequency with aspect ratio for both ends fixed condition

Table 12: Cantilever

\begin{tabular}{|l|l|l|l|l|l|l|l|l|l|l|}
\hline $\mathbf{L : W}$ & $\mathbf{1}$ & $\mathbf{2}$ & $\mathbf{3}$ & $\mathbf{4}$ & $\mathbf{5}$ & $\mathbf{6}$ & $\mathbf{7}$ & $\mathbf{8}$ & $\mathbf{9}$ & $\mathbf{1 0}$ \\
\hline $\mathbf{0 . 5}$ & 88.989 & 306.41 & 555.4 & 1030.7 & 1548.1 & 1953.1 & 2077.7 & 2860.3 & 3028.2 & 3344.8 \\
\hline $\mathbf{1}$ & 89.228 & 183.16 & 554.91 & 713.87 & 776.64 & 1273.3 & 1556.4 & 1696.6 & 1997.9 & 2233 \\
\hline $\mathbf{1 . 5}$ & 89.343 & 141.83 & 387.67 & 559.22 & 630.45 & 884.47 & 923.56 & 1371.9 & 1554.8 & 1625 \\
\hline $\mathbf{2}$ & 89.405 & 122.3 & 255.73 & 541.82 & 558.52 & 606.92 & 742.75 & 1006.2 & 1011.6 & 1428.2 \\
\hline $\mathbf{2 . 5}$ & 89.446 & 111.63 & 195.11 & 375.34 & 557.94 & 586.71 & 654.44 & 688.82 & 844.83 & 1058.2 \\
\hline
\end{tabular}

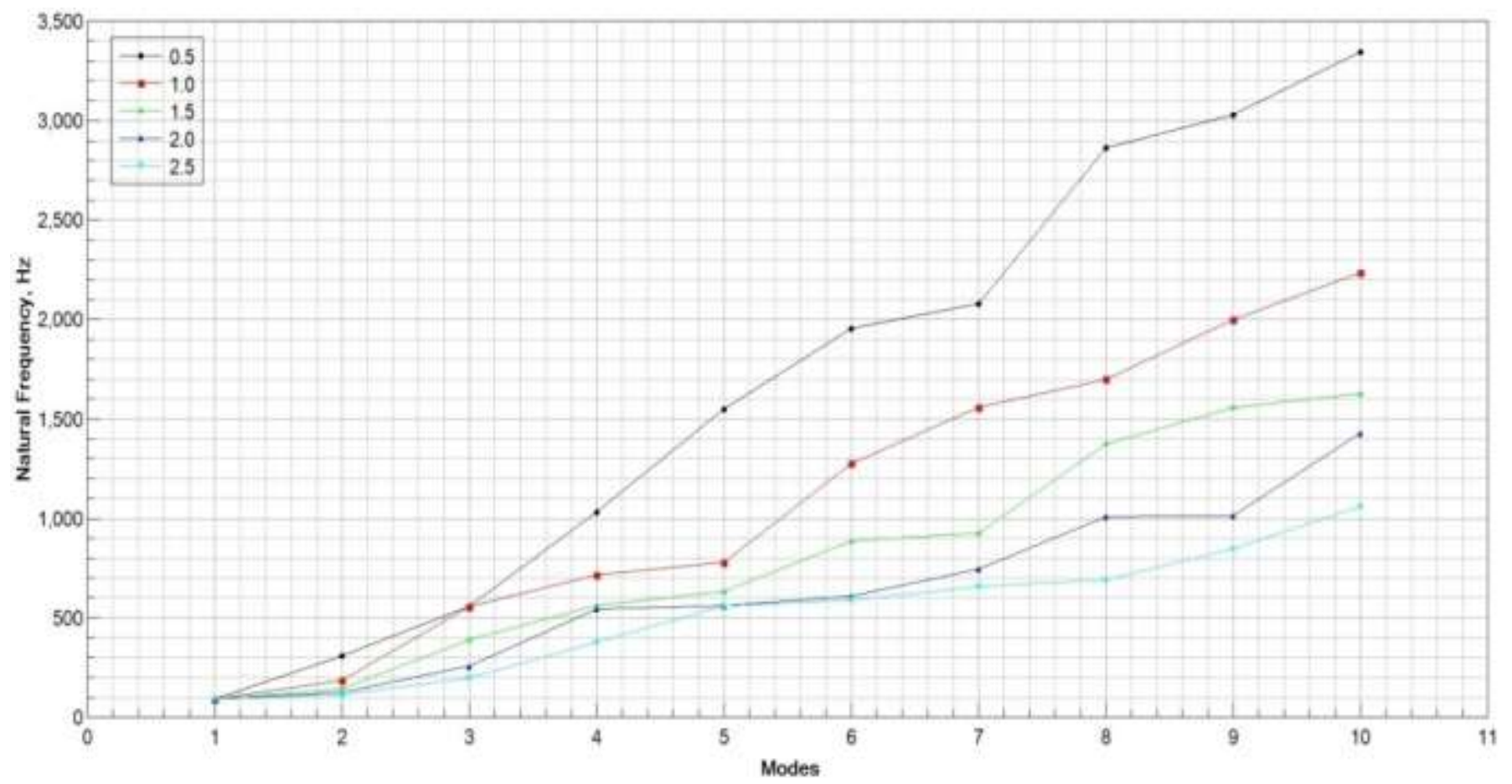

Fig 5: Variation of natural frequency with aspect ratio for cantilever condition 
Further the influence of Width:Thickness ratio on natural frequencies of composite plate was studied by considering plate width to be constant of $0.11 \mathrm{~m}$ and varying the thickness. The results obtained for Both End Fixed \& Cantilever boundary condition are listed in Table 13 and Table 14 respectively.
The obtained results are plotted in fig 6 and fig 7 for both ends fixed and cantilever boundary conditions and it was observed that the value of natural frequency decreased with increase in width: thickness ratio.

Table 13: Both Ends Fixed

\begin{tabular}{|l|l|l|l|l|l|l|l|l|l|l|}
\hline W: $\mathbf{T}$ & $\mathbf{1}$ & $\mathbf{2}$ & $\mathbf{3}$ & $\mathbf{4}$ & $\mathbf{5}$ & $\mathbf{6}$ & $\mathbf{7}$ & $\mathbf{8}$ & $\mathbf{9}$ & $\mathbf{1 0}$ \\
\hline $\mathbf{3 0}$ & 979.51 & 1043.4 & 1405.3 & 2359.3 & 2654.3 & 2740.3 & 3090.4 & 3879.5 & 3949.9 & 5093.7 \\
\hline $\mathbf{4 0}$ & 739.26 & 787.99 & 1062.2 & 1785.5 & 2018.2 & 2084.5 & 2353.1 & 2957.7 & 3000.4 & 3909 \\
\hline $\mathbf{5 0}$ & 592.96 & 632.29 & 852.74 & 1434.2 & 1624.6 & 1678.3 & 1895.7 & 2384.3 & 2414.3 & 3161 \\
\hline $\mathbf{6 0}$ & 587.24 & 625.11 & 836.49 & 1395.5 & 1609.2 & 1660.9 & 1869.8 & 2338.8 & 2341.3 & 3131.6 \\
\hline $\mathbf{7 0}$ & 423.7 & 451.98 & 609.89 & 1026.3 & 1164.5 & 1203.3 & 1360 & 1711.8 & 1730.2 & 2275.2 \\
\hline
\end{tabular}

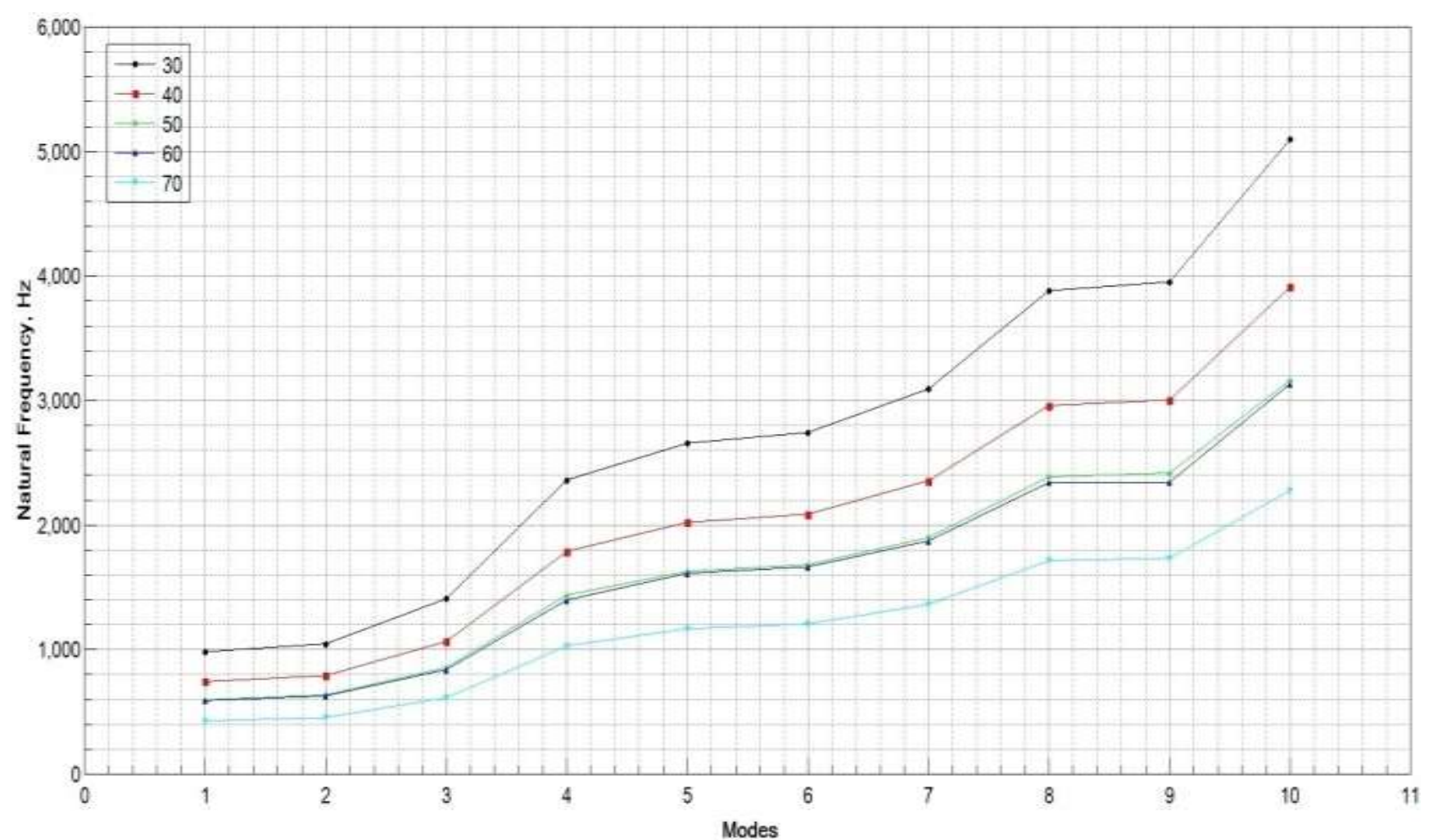

Fig 6: Variation of natural frequency with aspect ratio for both ends fixed condition

Table 14: Cantilever

\begin{tabular}{|l|l|l|l|l|l|l|l|l|l|l|}
\hline W: T & $\mathbf{1}$ & $\mathbf{2}$ & $\mathbf{3}$ & $\mathbf{4}$ & $\mathbf{5}$ & $\mathbf{6}$ & $\mathbf{7}$ & $\mathbf{8}$ & $\mathbf{9}$ & $\mathbf{1 0}$ \\
\hline $\mathbf{3 0}$ & 155.83 & 260.6 & 783.2 & 971.2 & 1117.2 & 1639.8 & 1908.1 & 2649.6 & 2672.8 & 2820.7 \\
\hline $\mathbf{4 0}$ & 116.96 & 196.07 & 589.3 & 732.26 & 842.87 & 1239.8 & 1439.6 & 2007.6 & 2026.1 & 2139.7 \\
\hline $\mathbf{5 0}$ & 93.572 & 157.07 & 472.07 & 586.89 & 676.07 & 995.63 & 1154.7 & 1613.8 & 1628.6 & 1720.6 \\
\hline $\mathbf{6 0}$ & 77.719 & 130.57 & 392.4 & 487.95 & 562.38 & 828.81 & 960.4 & 1344.2 & 1356.2 & 1433.3 \\
\hline $\mathbf{7 0}$ & 66.709 & 112.14 & 336.98 & 419.07 & 483.17 & 712.41 & 825.06 & 1155.7 & 1165.9 & 1232.3 \\
\hline
\end{tabular}




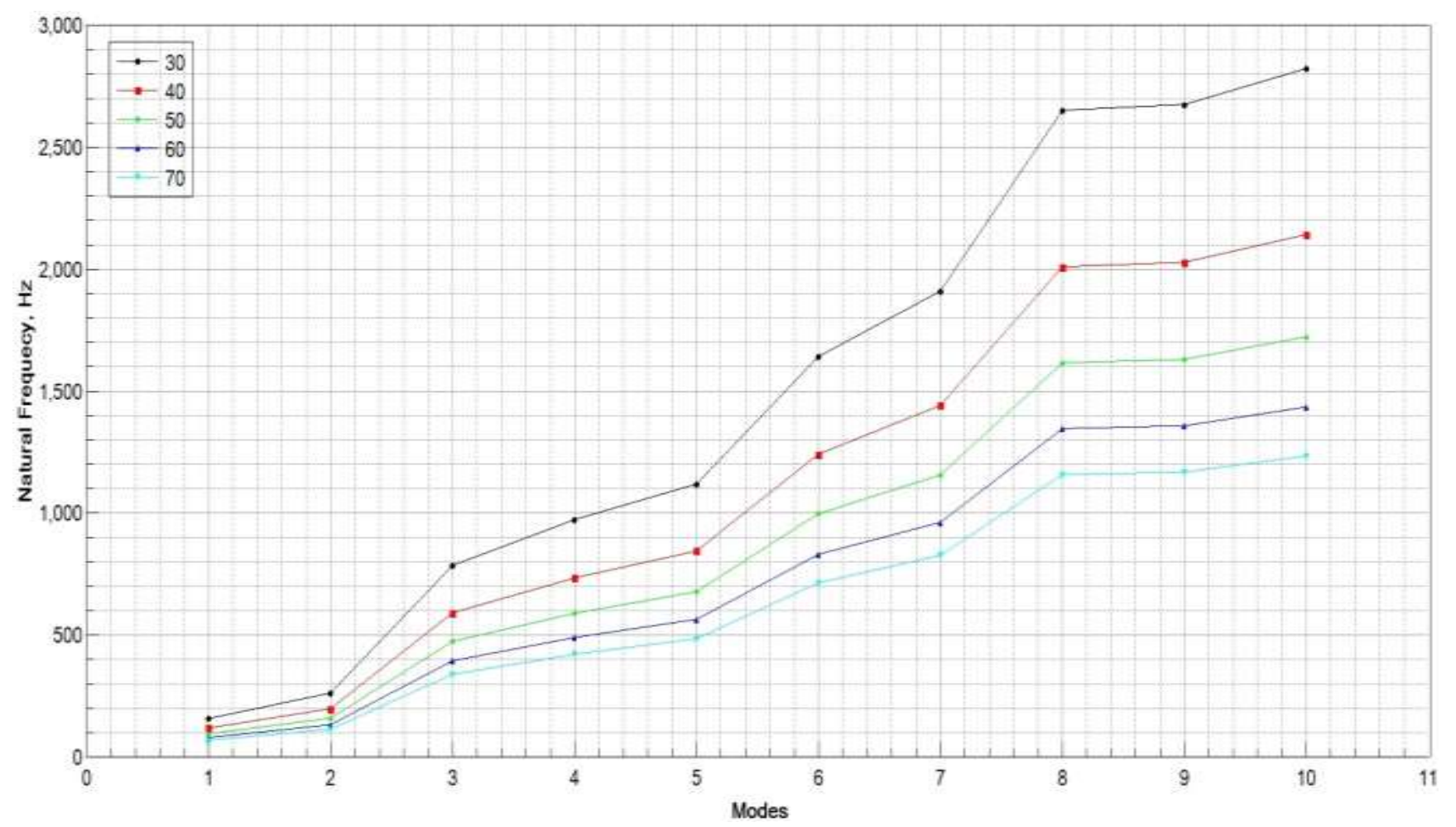

Fig 7: Variation of natural frequency with aspect ratio for cantilever condition

\section{COMPARISON}

Fig 8 shows the natural frequencies of all three materials for $[90 / 45]_{7}$ fiber orientation for both boundary conditions. And it can be seen that the Graphite-Epoxy material has the highest natural frequency among all three materials for most of the modes.

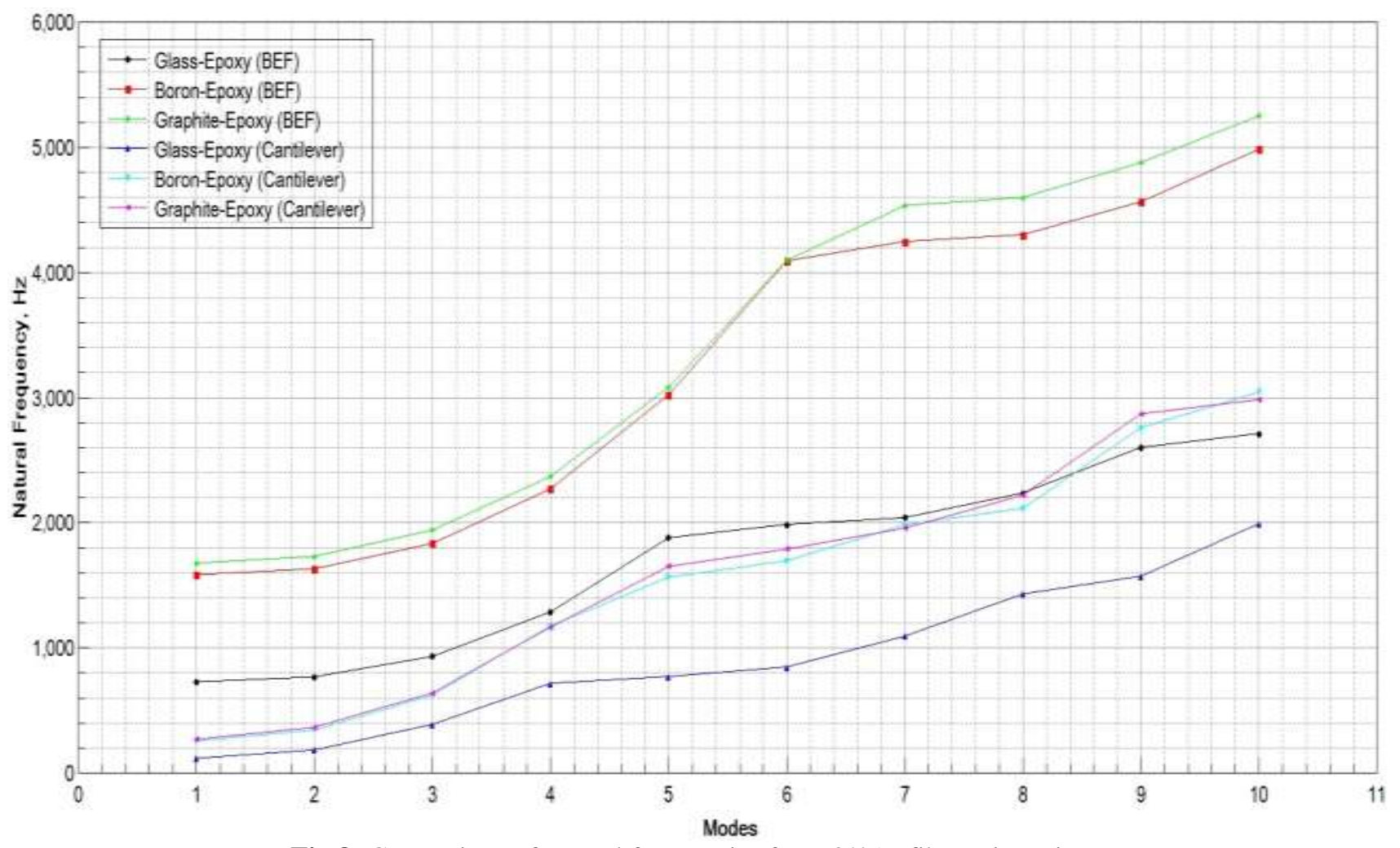

Fig 8: Comparison of natural frequencies for $[90 / 45]_{7}$ fiber orientation. 


\section{CONCLUSION}

In this work, modal analysis of composite plate without hole was carried out for three different materials to understand the variation of natural frequency by considering different fiber orientations subjected to varying boundary conditions using ANSYS simulation tool. It was observed that for all three materials natural frequency of composite plate was maximum for $[90 / 45]_{7}$ fiber orientation for most of the modes for both cantilever and both ends fixed boundary conditions. Among the three materials, Graphite-Epoxy was observed to have the maximum natural frequency while the least was for Glass-Epoxy for $[90 / 45]_{7}$ fiber orientation.

\section{REFERENCES}

[1] Dhanduvari Dinesh Kumar, Sri Hari M, Vinay Kumar Reddy “An Experimental and Numerical Approach to Free Analysis of Glass/Epoxy Laminated Composite Plates" Vol. 4 Issue (2015); 2278-0181.

[2] Itishree Mishra, Shishir Kumar Sahu, "An Experimental Approach to Free Vibration Response of Woven Fiber Composite Plates Under Free-Free Boundary Condition” Volume-1, Issue-2 (2012) 2231 $-5721$.

[3] Swapnil Chavan, "Study on Vibration Analysis of Composite Plate" Volume I Issue VIII, 2321-2705.

[4] Sharayu Ratnaparkhi, S. S. Samobat, "Vibration Analysis of Composite Plate" Vol.3, Issue.1 (2013), 2249-6645.

[5] Chaudhari, Y. R. Falak, "Vibration Analysis of Laminated Triangular Plate by Experimental and Finite Element Analysis" Volume 3, Issue 2, Part 2 (2015), 2091-2730.

[6] ChetanPatil, NileshFaranade, R. D Patil, "Vibration Analysis of Glass Epoxy Composite Plate with Variable No of Layers" Volume: 1 Issue: 3, 23497947.

[7] Nayak, Meher, Sahu, "Experimental and Numerical Study on Vibration and Buckling Characterstics of Glass-Carbon Epoxy Hybrid Composite Plates" Elsevier (2013).

[8] S. Parida, R. Dash, "A Study of Geometric Ratio and Fiber Orientation on Modal Characteristics of Composite Beam" Volume 2, Issue 8 (2014), 23494409.

[9] Ramu, S. Mohanty, "Study on Free Vibration Analysis of Rectangular Plate Structures Using Finite Element Method" Elsevier (2012), 2758-2766.

[10] Itishree Mishra, shishirshau, "Modal Analysis of woven Fiber Composite Plates with Different Boundary Conditions" Vol. 15, No. 1 (2015), 1540001 .

[11] Deshmukh P. V, Shrigandhi G. D., "Modal Analysis of Sandwich Panel", volume 2, issue-10, (2015), 2394-3696

[12] Autar K. Kaw "Mechanics of Composite Materials" $2^{\text {nd }}$ Edition - 2009

\section{BIOGRAPHIES}

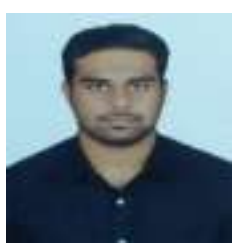

Sagar Umatar, Student, Mechanical Department, Jain College of Engineering.

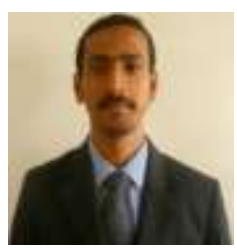

Vinayak Kallannavar (B. E, M.Tech), Assistant Professor, Mechanical Department, Jain College of Engineering.

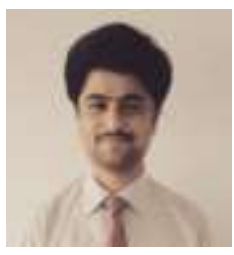

Vinayak Ratan (B.E, M.Tech), Assistant Professor, Mechanical Department, Jain College of Engineering.

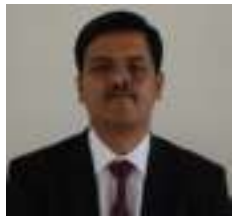

Dr. Anil Shirahatti (B.E, M.E, Ph.D), Professor, Mechanical Department, Jain College of Engineering. 